\title{
The immune status of patients with psoriasis vulgaris of blood-stasis syndrome and blood-dryness syndrome: a qualitative evidence synthesis
}

\author{
Jun-Tang Yan ${ }^{1}$, Qing-Guo Wang ${ }^{1}$, Xiao-Qian Liu ${ }^{1}$, Bin $\mathrm{Li}^{2,3}$, Yi Ru${ }^{2}$, Seokgyeong Hong ${ }^{2}$, Xiao-Ying Sun ${ }^{2}$,

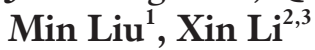 \\ ${ }^{1}$ School of Traditional Chinese Medicine, Beijing University of Chinese Medicine, Beijing, China; ${ }^{2}$ Department of Dermatology, Yueyang Hospital \\ of Integrated Traditional Chinese and Western Medicine, Shanghai University of Traditional Chinese Medicine, Shanghai, China; ${ }^{3}$ Institute of \\ Dermatology, Shanghai Academy of Traditional Chinese Medicine, Shanghai, China \\ Contributions: (I) Conception and design: M Liu, X Li; (II) Administrative support: QG Wang, B Li; (III) Provision of study materials or patients: XQ \\ Liu, Y Ru, S Hong, XY Sun; (IV) Collection and assembly of data: XQ Liu, Y Ru, S Hong, XY Sun; (V) Data analysis and interpretation: JT Yan, X \\ Li; (VI) Manuscript writing: All authors; (VII) Final approval of manuscript: All authors. \\ Correspondence to: Xin Li, MD, PhD. Department of Dermatology, Yueyang Hospital of Integrated Traditional Chinese and Western Medicine, \\ Shanghai University of Traditional Chinese Medicine, Shanghai, China. Email: 13661956326@163.com. Min Liu, PhD. School of Traditional \\ Chinese Medicine, Beijing University of Chinese Medicine, Beijing, China. Email: liumin78@126.com.
}

Background: Chinese medicine (CM) classifies psoriasis vulgaris into three syndromes: blood-heat syndrome (BHS), blood-stasis syndrome (BSS), and blood-dryness syndrome (BDS). The levels of several immunological serum markers in BHS have been established. We aimed to investigate the immune status of patients with psoriasis vulgaris of BSS and BDS.

Methods: Seven databases were searched, covering nearly 40 years. Fifteen studies including 957 individuals (386 patients with psoriasis vulgaris of BSS, 233 patients with BDS, and 338 healthy controls) were identified. Differences in interleukin (IL) levels between subjects and controls were pooled as mean differences (MDs) with 95\% confidence intervals (CI) using a random-effects model.

Results: For BSS, interferon (IFN)- $\gamma$ (MD 3.85, 95\% CI: 1.27 to 6.44), tumor necrosis factor (TNF)- $\alpha$ (MD 1.71, 95\% CI: 0.70 to 2.72), IL-4 (MD 7.66, 95\% CI: 4.67 to 10.65 ), IL-17 (MD 5.06, 95\% CI: 0.28 to 9.85), IL-6 (MD 99.34, 95\% CI: 45.84 to 152.84), and IL-22 (43.88, 95\% CI: 28.17 to 59.59) levels were significantly higher in patients than in controls, while pooled IL-10 levels were lower in patients (MD -10.33, 95\% CI: -12.03 to -8.63). The MD in IL-8 levels between cases and controls was not significant. Subjects with BDS showed higher levels of IFN- $\gamma$ (MD 2.33, 95\% CI: 0.22 to 4.45), TNF- $\alpha$ (MD 2.33, 95\% CI: 1.26 to 3.40), and IL-23 (MD 46.18, 95\% CI: -7.60 to 99.97) and lower levels of IL-4 levels (MD $-2.47,95 \%$ CI: -4.78 to -0.15 ) than did controls. The MDs in IL-17, IL-6, and IL-8 levels were not statistically significant.

Conclusions: Our pooled analysis suggests that the levels of several ILs are specifically altered in BSS and BDS. Larger, well designed, controlled studies are needed to confirm these results and fully clarify these effects.

Keywords: Psoriasis vulgaris; Chinese medicine (CM); blood-stasis syndrome (BSS); blood-dryness syndrome (BDS); immune status

Submitted Oct 31, 2019. Accepted for publication Apr 07, 2020.

doi: $10.21037 /$ apm-19-432

View this article at: http://dx.doi.org/10.21037/apm-19-432 


\section{Introduction}

Psoriasis is a chronic, immune-mediated, systemic disorder that affects approximately $0.9-8.5 \%$ of the population worldwide $(1,2)$. Psoriasis is characterized by sustained inflammation and epidermal hyperplasia, ultimately leading to the formation and persistence of lesions, which are commonly located on the scalp, elbows, knees, umbilicus, and waist. Psoriasis impacts patients both physically and psychologically, resulting in the decline of physical and mental function $(3,4)$. Moderate to severe psoriasis is associated with an increased risk of comorbidities, including cardiovascular diseases (5), hypertension (6), chronic obstructive pulmonary disease (7), hyperuricemia (8), and depression (9), leading to marked impairment of the quality of life. The pathogenesis of this disease is not fully understood, but abnormal growth of keratinocytes and infiltration of inflammatory cells are involved (10). A considerable number of inflammatory cytokines are involved in psoriasis lesions on the skin, and the serum concentrations of a subset of these are also associated with disease severity. Specifically, interleukin (IL)-1, IL-2, IL-4, IL-6, IL-8, IL-10, IL-12, IL-17, IL-20, IL-22, IL-23, IL-36, and tumor necrosis factor (TNF)- $\alpha$ are the main ILs believed to be closely related to the pathogenesis, and they are considered as therapeutic targets in pivotal clinical trials (11).

Currently, oral agents (methotrexate, acitretin, and cyclosporine), topical agents (topical corticosteroids, tarbased preparations, dithranol, vitamin D analogues, salicylic acid, and topical retinoids), and a growing repertoire of biologic agents (infliximab, adalimumab, etanercept, ustekinumab, etc.) are being used as pharmacological treatments. However, $52.3 \%$ of the patients with psoriasis report dissatisfaction with the medical treatment, which often does not meet their expectations and is marred by adverse effects. Not surprisingly, $51 \%$ of the patients with psoriasis were reported to opt for alternative therapies (12), including acupuncture (13), cupping (14), herbal medicine (15), fish oil (16), total glucosides of paeony (17), and Tripterygium wilfordii Hook. f. (18).

Chinese medicine $(\mathrm{CM})$ is an alternative therapy based on more than 2,500 years of Chinese medical practice, and it is widely used for the treatment of dermatological conditions based on its unique and systemic theory (19). CM has been an important clinical method for treating psoriasis in China and is characterized by a holistic view. CM differentiates psoriasis vulgaris into three main syndromes: blood-heat syndrome (BHS), blood-stasis syndrome (BSS), and blood-dryness syndrome (BDS) (19). These categories respectively correspond to three principles of therapeutic intervention: clearing heat and cooling blood, promoting blood circulation to dissipate blood stasis, and adding moisture to reduce blood dryness (19-21).

Recently, CM has been influenced by modern Western medicine and has gradually become the focus of research because of its promising effects. As the most common syndrome during the active period of psoriasis vulgaris, BHS has been studied earlier and more intensely than have the other syndromes from a Western medical perspective. A previous study observed significantly elevated serum levels of interferon (IFN)- $\gamma$, IL-17, IL-23, and TNF- $\alpha$, and significantly decreased levels of IL-4 and IL-10 in BHS (22). However, the immune status of patients with psoriasis vulgaris of BSS and BDS has not been investigated in detail. Here, we performed a meta-analysis to investigate several well-known ILs (IFN- $\gamma$, IL-4, IL-6, IL-8, IL-10, IL-17, IL-22, IL-23, and TNF- $\alpha$ ) in patients with psoriasis vulgaris of BSS and BDS.

\section{Methods}

\section{Eligibility criteria}

Inclusion and exclusion criteria were determined before the literature search was conducted. We included human studies comparing patients with psoriasis vulgaris of BSS and BDS with healthy controls and measuring one or more of the following immunological markers in the serum: IFN- $\gamma$, IL-4, IL-17, IL-23, IL-6, TNF- $\alpha$, IL-10, IL-22, and IL-8. If several studies reported results from the same study population, the most complete report was included. Case reports and letters were excluded.

\section{Data sources and searches}

To identify relevant studies on psoriasis vulgaris of BSS and BDS that included the assessment of immunological markers, three reviewers (JT Yan, XQ Liu, Y Ru) systematically searched in MEDLINE, Embase, the Cochrane Central Register, the China National Knowledge Infrastructure database, the Chinese Scientific Journals Full Text Database, the Wanfang Data Knowledge Service Platform, and the Chinese Biomedical Literature Service System using the following search terms: psoriasis, BSS, BDS, IFN- $\gamma$, IL-4, IL-17, IL-23, IL-6, TNF- $\alpha$, IL-10, IL-22, and IL-8. Papers published in English or Chinese 


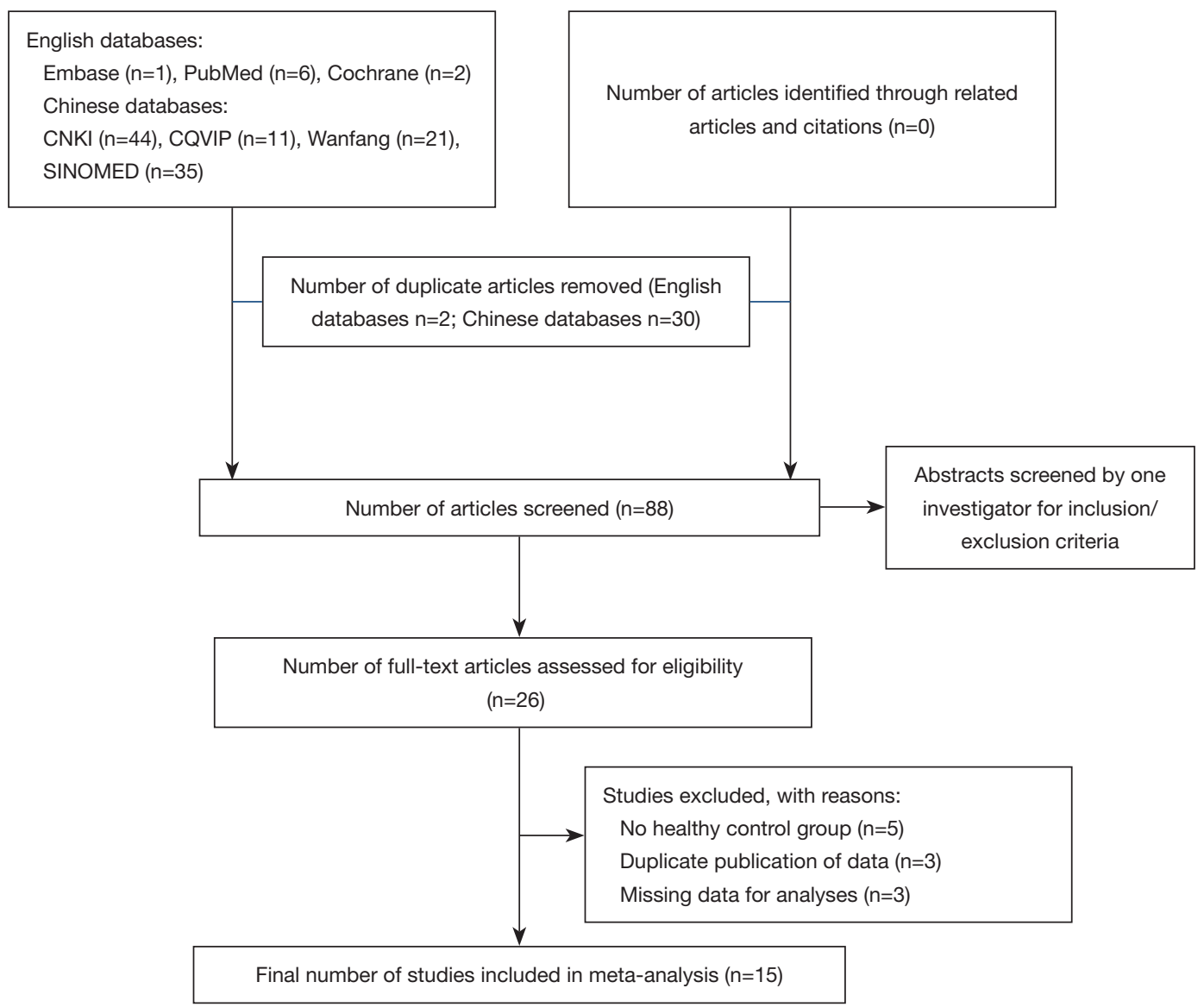

Figure 1 Flowchart of the literature search and study selection process. CNKI, China National Knowledge Infrastructure database; CQVIP, Chinese Scientific Journals Full Text Database; SINOMED, Chinese Biomedical Literature Service System.

and dated from January 1980 to March 2020 were included in this study.

\section{Study selection}

We screened all titles and abstracts to identify and select the analyses comparing immunological marker profiles of patients with psoriasis vulgaris of BSS and/or BDS with those of controls. No limitations were set in terms of the study design and the age, sex, and nationality of participants. The inclusion criteria were as follows: (I) human studies, (II) original data, (III) a healthy control group, and (IV) reporting of means and confidence intervals (CIs) for immunological serum markers. We identified 120 articles in the initial search (Figure 1). After removing 32 duplicate articles and reading 88 individual abstracts, we identified 26 original studies eligible for inclusion criteria assessment. After reviewing the full text of these 26 studies, we excluded
11 articles for the following reasons: no healthy control group, duplicate publication of data, and missing analysis data. Finally, 15 studies that met the inclusion criteria were analyzed (23-37). A flowchart of the search process is shown in Figure 1.

\section{Data extraction and quality assessment}

Three reviewers independently collected the following descriptive data for each included study: (I) first author, (II) study characteristics (i.e., year and country), (III) characteristics of participants [i.e., mean age, male ratio, number of cases and controls, course, and mean Psoriasis Area and Severity Index (PASI) score for cases], and (IV) outcome characteristics (i.e., mean levels of immunological serum markers in psoriasis vulgaris BSS and BDS along with their standard deviations, and whether results were obtained from the primary analysis of the study or were 
adjusted for comorbidities).

The Newcastle-Ottawa Scale (38) was used to assess the quality of the studies. It categorizes studies using three dimensions, namely selection, comparability, and exposure for case-control studies and selection, comparability, and outcome for cohort studies. Selection, comparability, exposure, and outcome include four, one, three, and three items, respectively.

\section{Data synthesis and analysis}

The primary outcome was the difference in the mean serum levels of immunological markers between patients with psoriasis vulgaris of BSS or BDS and healthy controls (Table 1). The degree of heterogeneity between studies was assessed using the $\mathrm{I}^{2}$ test. An $\mathrm{I}^{2}$ value of $>50 \%$ was considered to indicate substantial heterogeneity, in which case DerSimonian and Laird random-effects models were used to compute the global MD. When the betweenstudy heterogeneity was not substantial $\left(\mathrm{I}^{2}<50 \%\right)$, a fixedeffects model was considered suitable. The methods and findings of the present review have been reported in accordance with the meta-analysis of Observational Studies in Epidemiology group guidelines and checklist (39). The Cochrane Collaboration software Review Manager 5.2 was used to perform the meta-analysis (http://ims.cochrane.org/revman).

\section{Results}

\section{Study selection}

From a total of 120 titles, the full text of 26 potentially relevant studies was reviewed to confirm their eligibility. Among these 26 studies, 11 were excluded, including five with no healthy control group, three with duplicated publication of data, and three with missing analysis data. In total, 15 trials met the inclusion criteria (Figure 1).

\section{Study characteristics}

The 15 selected studies included data for 957 individuals (386 patients with psoriasis vulgaris of BSS, 233 patients with BDS, and 338 healthy controls). All 15 studies were conducted in China; 13 were published in Chinese and two in English. The age and male ratios in the psoriasis vulgaris and healthy control groups were comparable among studies (Table 2). Moreover, the age, male ratios, PASI score, and course were also comparable between patients with psoriasis vulgaris of BSS and those with psoriasis vulgaris of BDS. In total, $66.67 \%$ studies reported PASI scores, and $40 \%$ patients were from studies reporting a mean PASI score of $>10$. This indicated that some of the studies included patients with severe disease. The reported NewcastleOttawa Scale scores were between 4 and 6, corresponding to medium quality, as shown in Table 3. Specifically, two of the studies received four stars $(26,27)$, four studies received five stars $(23,24,35,36)$, and the remaining studies received six stars.

\section{Outcomes}

With the exception of IL-23 levels in subjects with psoriasis vulgaris of BSS, meta-analysis of the results for all markers revealed significant between-study heterogeneity $\left(\mathrm{I}^{2}>75 \%\right)$; thus, a random-effects model was used.

For subjects with psoriasis vulgaris of BSS, the MD in studies analyzing IFN- $\gamma$ was 3.85 (95\% CI: 1.27 to 6.44), indicating a significant difference in serum IFN- $\gamma$ levels between 156 patients and 148 controls (Figure 2A). Seven studies reported plasma IL-4 levels in 189 patients and 142 controls. Figure $2 B$ shows that the IL-4 level was significantly higher in patients than in controls, with a pooled MD of 7.66 (95\% CI: 4.67 to 10.65). Six studies (including 152 patients and 125 controls) showed a significant, positive MD for the IL-17 level (5.06, 95\% CI: 0.28 to 9.85 ; Figure $2 C$ ). The MD in the IL-23 level between 45 patients and 25 controls was not statistically significant (MD 46.18; 95\% CI: -7.60 to 99.97; Figure 2D). Pooling of IL-6 levels revealed significantly higher values for 165 patients than for 101 healthy controls (MD 99.34; 95\% CI: 45.84 to 152.84; Figure 2E). A total of six articles including 206 patients and 162 controls evaluated TNF- $\alpha$, which was significantly higher in patients than in controls (MD 1.71, 95\% CI: 0.70 to 2.72; Figure 2F). Meanwhile, the IL-10 level was significantly lower in 64 patients than that in 71 controls (MD -5.26, 95\% CI: -9.53 to -0.99; Figure $2 G$ ). Three studies (including 66 patients and 46 controls) evaluated IL-22, and pooled analysis revealed significantly higher values for patients than for controls (MD 43.88, 95\% CI: 28.17 to 59.59; Figure $2 \mathrm{H}$ ). With regard to IL-8 levels, the MD between 133 patients and 110 healthy controls was not significant (MD 0.02; $95 \%$ CI: -0.99 to 1.03 ; Figure 2 ).

For subjects with psoriasis vulgaris of BDS, the mean IFN- $\gamma$ level across studies was significantly higher in 104 patients than in 112 controls (MD 2.33; 95\% CI: 0.22 to 4.45 ; Figure $3 A$ ), while the IL-4 level was lower in 62 


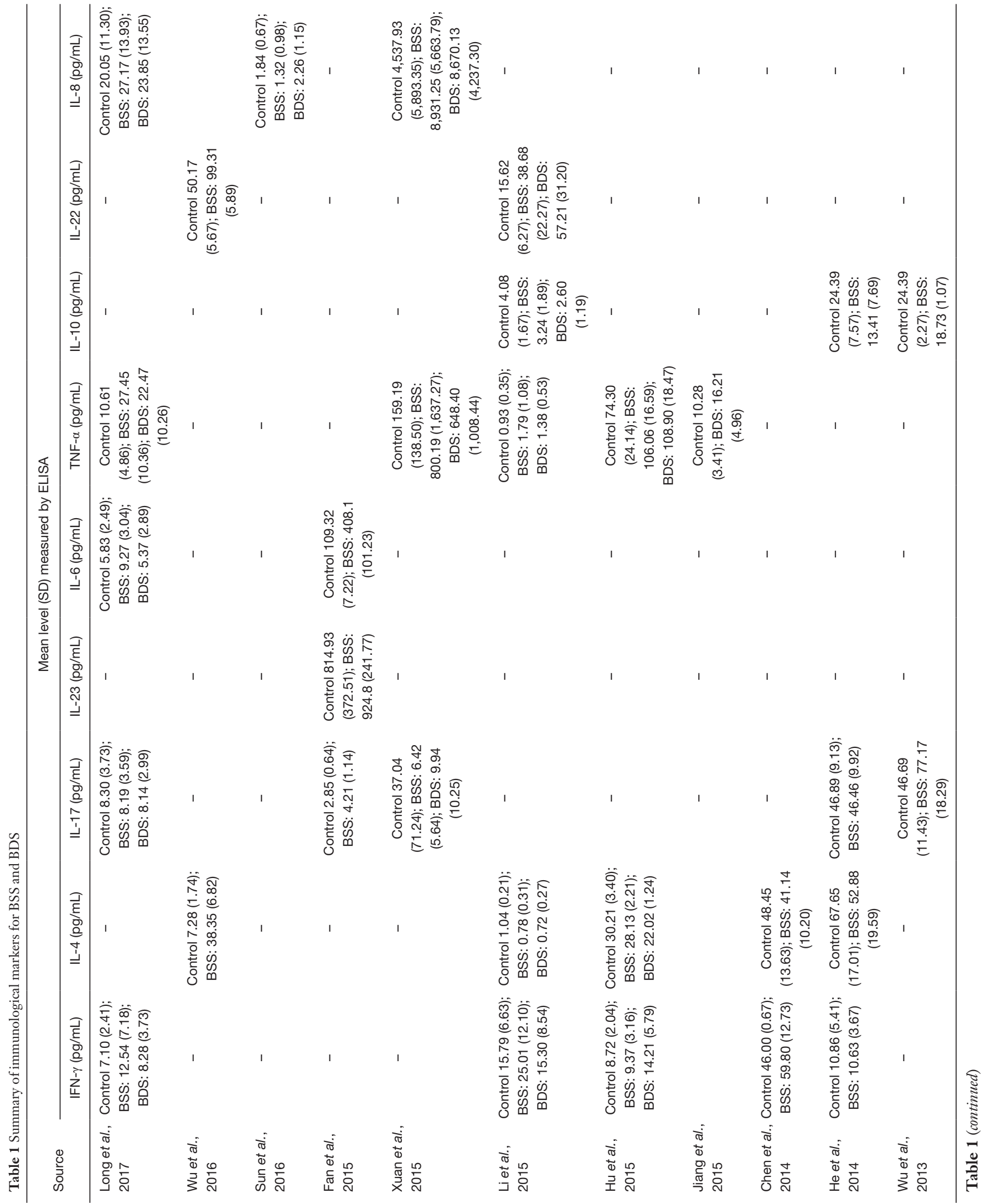




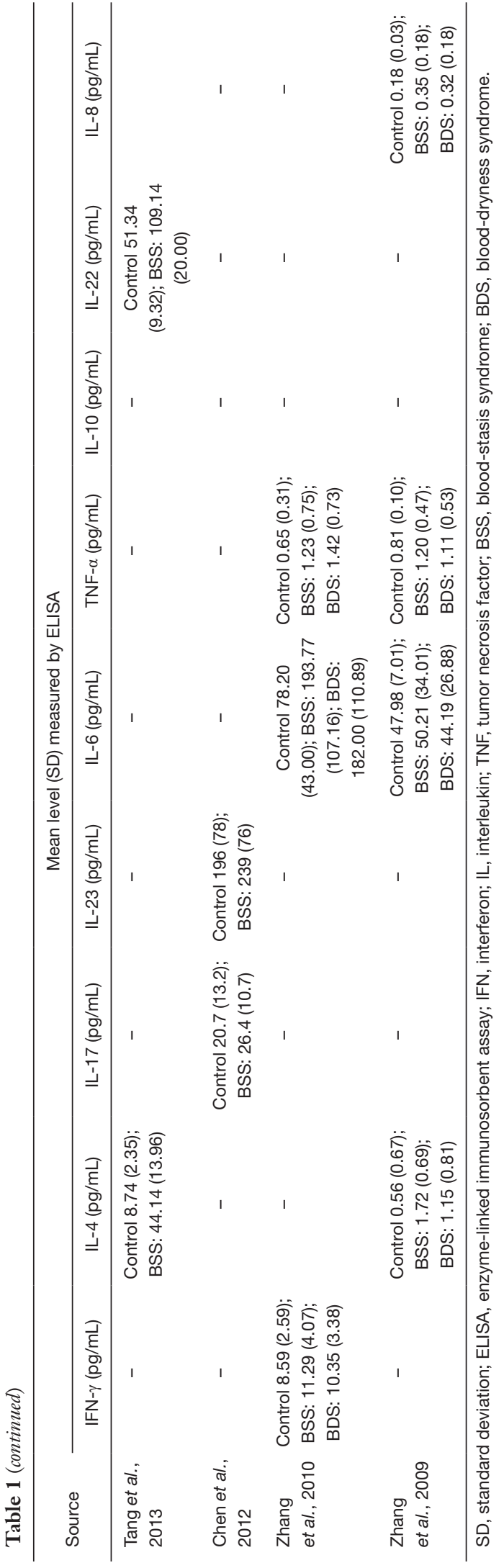

patients than in 81 controls, with a combined MD of -2.47 (95\% CI: -4.78 to -0.15 ; Figure 3B). IL-17 levels showed a non-significant MD between 51 patients and 50 healthy controls (MD -8.84; 95\% CI: -33.52 to 15.83 ; Figure 3C). The combined MD for IL-6 levels in 80 patients and 91 healthy controls was positive but not statistically significant (MD 18.93; 95\% CI: -6.72 to 44.58 ; Figure 3D). The MD in TNF- $\alpha$ levels was 2.33 (95\% CI: 1.26 to 3.40), indicating a significant difference between 222 patients and 192 controls (Figure 3E). Four studies (including 81 patients and 110 controls) showed a non-significant MD in IL-8 levels (MD 0.33, 95\% CI: -0.57 to 1.22 ; Figure $3 F$ ).

The MDs in immunological markers between BHS and controls, BSS and controls, and BDS and controls are shown in Table 4.

\section{Discussion}

\section{Summary of evidence}

This review involved a systematic assessment of 15 studies originating from China, which measured the main ILs in patients with psoriasis vulgaris of BSS and BDS. The pooled data showed that serum IFN- $\gamma$, IL-4, IL-17, IL-6, TNF- $\alpha$, and IL-22 levels were higher in patients with BSS than in controls, while serum IFN- $\gamma$ and TNF- $\alpha$ levels were higher in patients with BDS than in controls. Pooled IL-10 and IL-4 levels were significantly lower in patients with BSS and BDS, respectively, than in controls. These ILs and various cell types are involved in the pathogenesis of psoriasis vulgaris of BHS, BSS, and BDS through several mechanisms. The first mechanism involves $\mathrm{T}$ helper (Th) $1 / \mathrm{Th} 2$ homeostasis. The pathogenesis of psoriasis involves the immune responses of both Th1 and Th2 cells, with the Th1/Th2 balance showing various degrees of skewing in different psoriasis syndromes, as shown by measurement of specific cytokines and ratios of Th1/Th2 cells (including IFN- $\gamma$, TNF- $\alpha$, IL-4, IL-13, etc.). The second mechanism involves Th17/regulatory $\mathrm{T}$ (Treg) cell balance. Treg cells usually suppress other pathogenic $\mathrm{T}$ cells, such as Th1 and Th17 cells, in psoriasis, thus inducing a balance between Tregs and Th17 cells, regulated by a combination of IL-23, IL-6, IL-10, TGF- $\beta$, IL-35, and IL-17, as well as other $\mathrm{T}$ cell populations. The third mechanism involves the IL-23/Th17 axis. IL-23 plays a pivotal role in the survival and proliferation of Th17 cells, contributing to keratinocyte hyperproliferation and thus facilitating the development of psoriasis. In turn, the activated Th17 


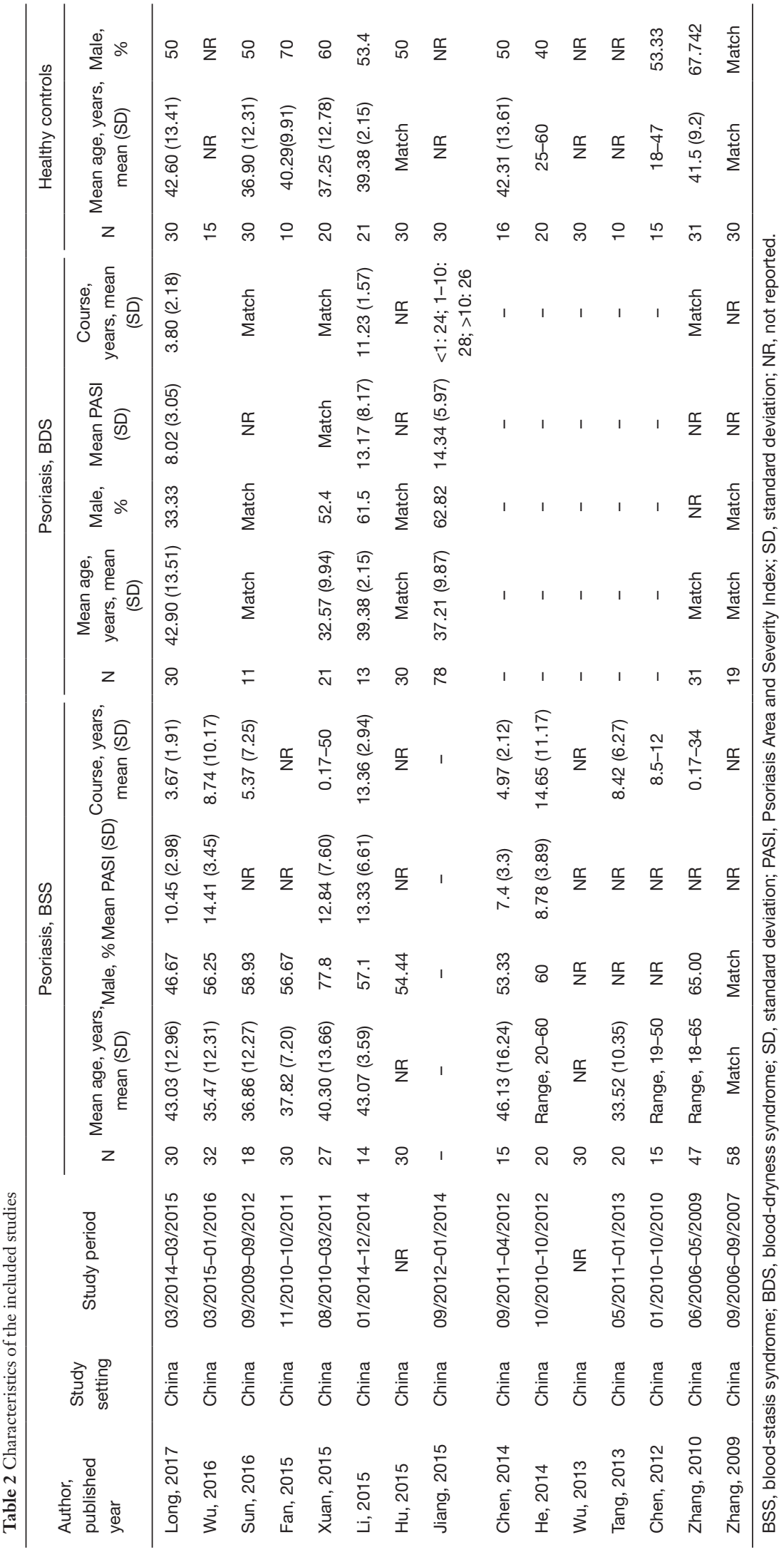


Table 3 Newcastle-Ottawa Scale (NOS) quality assessment table

\begin{tabular}{|c|c|c|c|c|}
\hline Study, year & Selection & Comparability & Exposure/outcome & Overall star rating \\
\hline Wu, 2016 & ++ & & +++ & 5 \\
\hline Sun, 2016 & + & + & +++ & 5 \\
\hline Fan, 2015 & + & ++ & +++ & 6 \\
\hline Li, 2015 & + & ++ & +++ & 6 \\
\hline $\mathrm{Hu}, 2015$ & ++ & + & +++ & 6 \\
\hline Jiang, 2015 & ++ & + & +++ & 6 \\
\hline Chen, 2014 & + & ++ & +++ & 6 \\
\hline Tang, 2013 & ++ & & ++ & 4 \\
\hline Chen, 2012 & + & ++ & +++ & 6 \\
\hline Zhang, 2010 & & ++ & +++ & 5 \\
\hline Zhang, 2009 & + & + & +++ & 5 \\
\hline
\end{tabular}

A star system was used to allow a semiquantitative assessment of study quality. A study could be awarded a maximum of one star for each numbered item within the selection and exposure categories. A maximum of two stars could be awarded for comparability. The NOS total score ranges from zero to nine stars. We considered high-quality studies those that achieved seven or more stars; medium-quality studies those that achieved four to six stars; and poor-quality studies those that achieved fewer than four stars.

cells enhance the inflammatory response of keratinocytes, resulting in a positive feedback loop around the IL-23/ Th17 axis. IL-22, originating from Th17 and Th22 cells, induces IL-23-mediated keratinocyte hyperproliferation. The fourth mechanism involves other pathways and signaling molecules, including $\gamma \delta \mathrm{T}$ cells, CD8+ T cells, macrophages, neutrophils, and their related antimicrobial peptides, cytokines, and chemokines such as CCL20 and CXCLs (including IL-8) secreted by keratinocytes, which act as chemoattractant to amplify the immune response in psoriasis. A simplified model depicting the role of the inflammatory cytokines in psoriasis vulgaris of BHS, BSS, and BDS is shown in Figure 4.

\section{Possible rationales}

According to $\mathrm{CM}$ theories, there are three basic psoriasis vulgaris syndromes: BHS (53.8\% cases), BDS (27.4\%), and BSS (18.1\%) (40). Patients usually present with BHD at the beginning of the active stage, which may be converted to BSS or BDS later. The effects of the multiple cell types involved in psoriasis, including effector $\mathrm{T}$ cells, dendritic cells, neutrophils, and macrophages, are mediated by a complex network of cytokines and their interactions. IFN- $\gamma$ and TNF- $\alpha$ act on keratinocytes, leading to their activation and proliferation. It has been demonstrated that IFN- $\gamma$ is a useful biomarker for psoriasis disease activity because of its positive correlation with PASI (41). This explains why the MDs in IFN- $\gamma$ and TNF- $\alpha$ levels between BHS and controls were $>10$, as shown in Figure 4 and Table 4. IL-4 improves the disease not only by promoting Th2 polarization, but also by directly inhibiting inflammatory cytokines in epidermal cells (42). Interestingly, the combined MD for IL-4 levels indicated decreased levels in the BHS and BDS groups and increased levels in the BSS group, relative to the respective control groups. The altered balance between IL-23 and Th17 cells is closely related to IL-17 and IL-17secreting cells (Th17 cells, $\gamma \delta$ T cells, neutrophils, etc.), and IL-23 plays a key role in the survival and proliferation of Th17 cells (43). Surprisingly, IL-23 was associated with a non-significant MD between patients with psoriasis vulgaris of BSS and controls. However, this result is consistent 

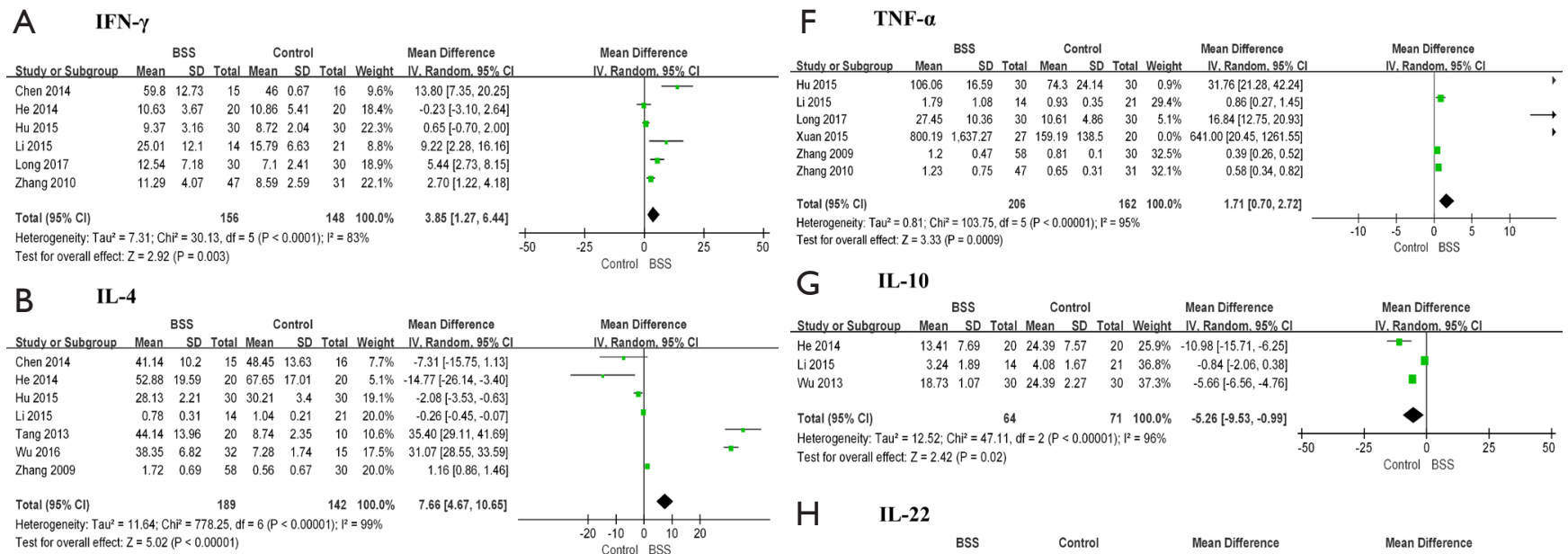
Test for overall effect: $Z=5.02(P<0.00001)$
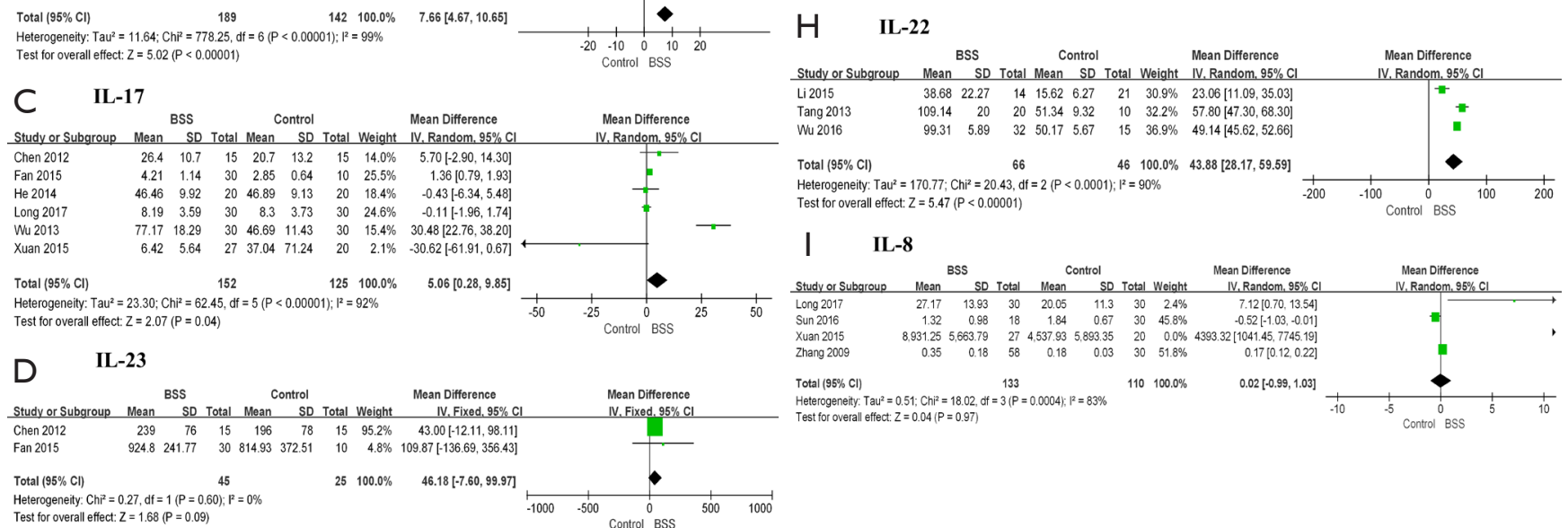

Heterogeneity: $C h^{2}=0.27$, df $=1(P=0.50): R=0.0$

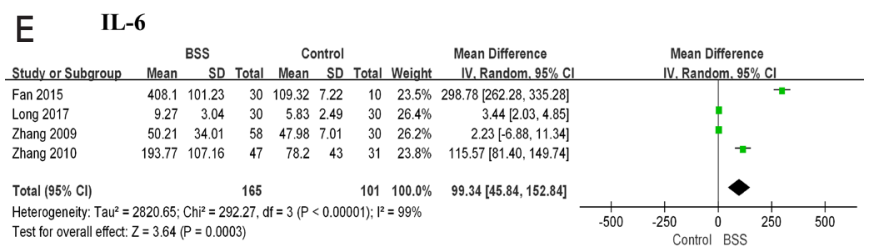

Figure 2 Meta-analysis of serum IFN- $\gamma$, IL-4, IL-17, IL-23, IL-6, TNF- $\alpha$, IL-10, IL-22, and IL-8 levels in patients with psoriasis vulgaris of BSS. The MD between psoriasis patients and controls is shown for each marker. The point estimate is represented as the center of each green square, and the statistical size is proportional to the area of the square. Horizontal lines indicate $95 \%$ CI. The subtotal and total MDs (diamonds) were calculated using random-effects models. SD, standard deviation; IFN- $\gamma$, levels of interferon $\gamma$; TNF- $\alpha$, tumor necrosis factor $\alpha$; IL, interleukin; MD, mean difference; BSS, blood-stasis syndrome.

with the distribution of BSS, which is most common in the resting and regressive stages. IL-22, derived from Th17 and Th22 cells, induces IL-23-mediated keratinocyte hyperproliferation. The combination of IL-22 and IL-17 inhibits the differentiation of keratinocytes and increases their proliferation and activity. Treg cells play an important role in maintaining homeostasis and may cause local inhibition of other immune cells, including Th17 cells. IL-6 is required for the differentiation of Treg cells, hindering the activation and proliferation of Th17 cells. IL-10 is expressed by mature Treg cells, which help in limiting psoriasis and preventing autoimmune diseases (44).

$\mathrm{CM}$ is an effective therapy for psoriasis, as demonstrated by a number of previous studies (45-48). Chinese herbal compounds, monomers, and efficient components are often used to directly or indirectly regulate immunity by acting on 
A IFN- $\gamma$

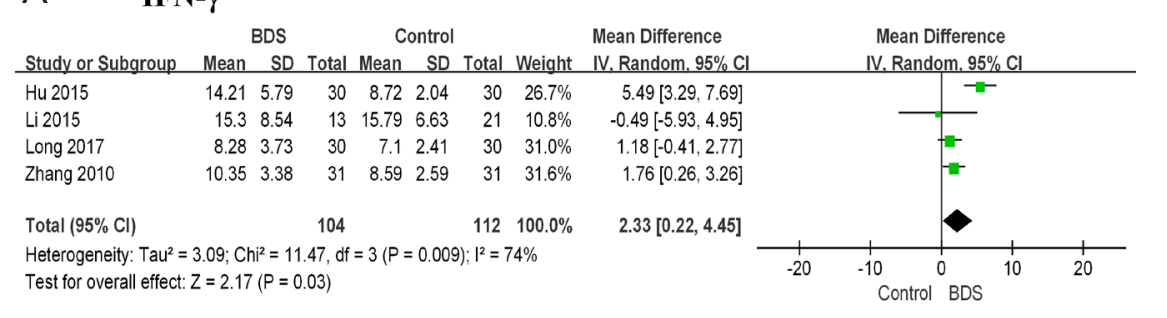

B IL-4

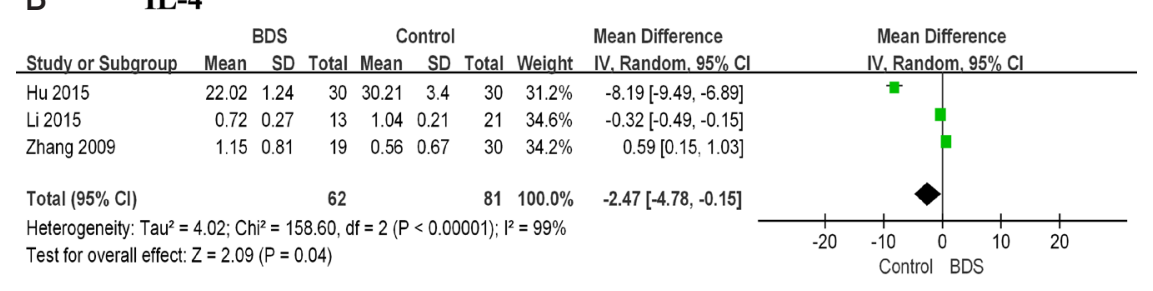

\section{IL-17}

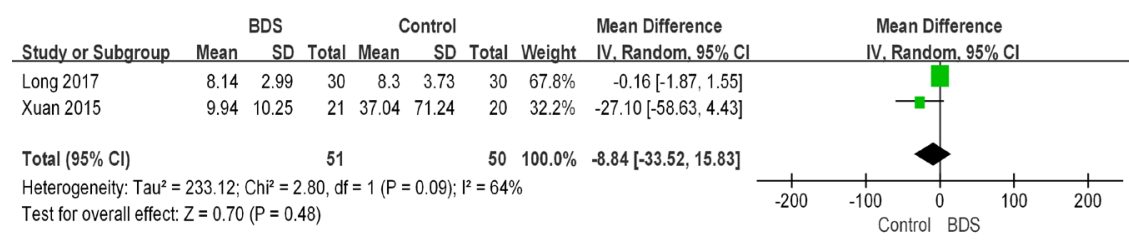

D IL-6

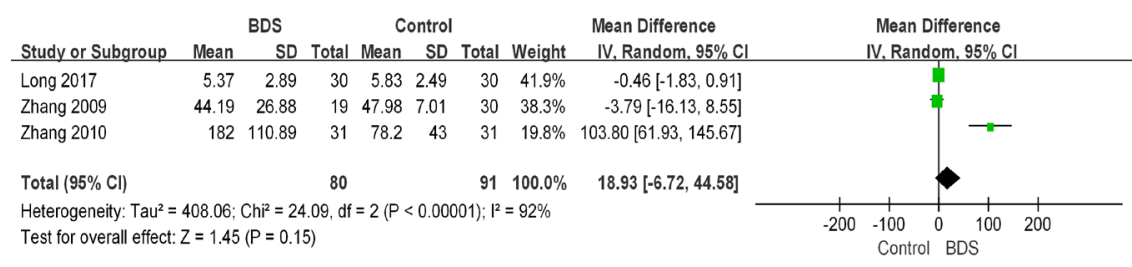

\section{E TNF- $\alpha$}

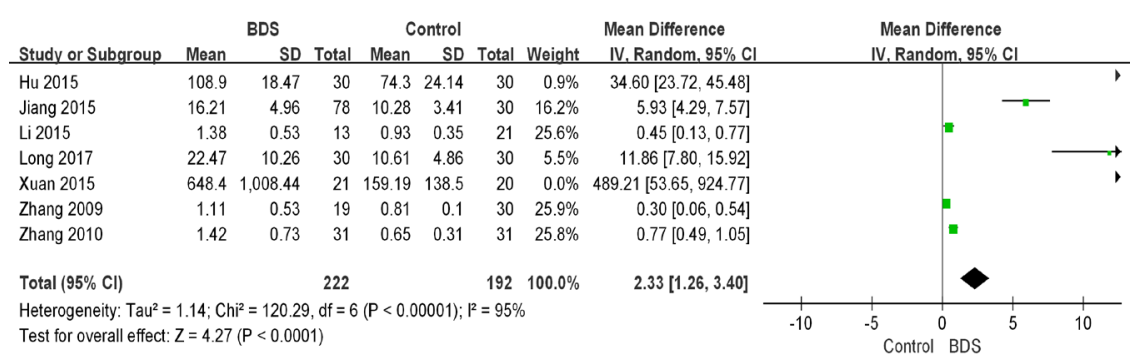

F IL-8

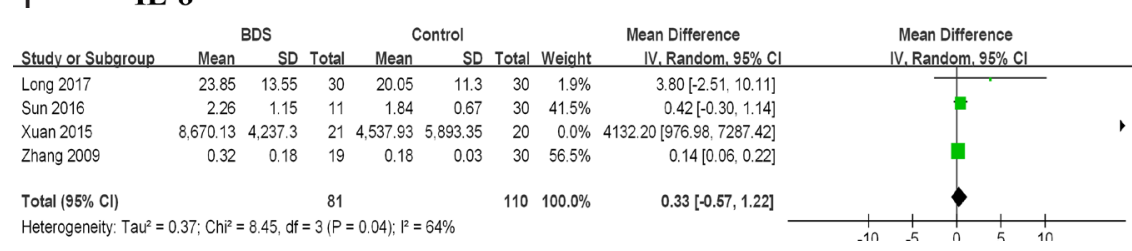

Figure 3 Meta-analysis of IFN- $\gamma$, IL-4, IL-17, IL-6, TNF- $\alpha$, and IL-8 levels in patients with psoriasis vulgaris of BDS. The MD between psoriasis patients and controls is shown for each marker. The point estimate is represented as the center of each green square, and the statistical size is proportional to the area of the square. Horizontal lines indicate $95 \%$ confidence intervals. The subtotal and total MDs (diamonds) were calculated using random-effects models. SD, standard deviation; IFN- $\gamma$, levels of interferon $\gamma$; IL, interleukin; TNF- $\alpha$, tumor necrosis factor $\alpha$; BSS, blood-stasis syndrome; MD, mean difference. 
Table 4 Mean difference in immunological markers between blood-heat syndrome and controls, BSS and controls, and BDS and controls

\begin{tabular}{lccc}
\hline Markers & BHS vs. control, MD $(95 \% \mathrm{Cl})$ & BSS vs. control, MD $(95 \% \mathrm{Cl})$ & BDS vs. control, MD (95\% Cl) \\
\hline IFN- $\gamma$ & $24.90(12.36,37.43)^{\star}$ & $3.85(1.27,6.44)^{\star}$ & $2.33(0.22,4.45)^{\star}$ \\
TNF- $\alpha$ & $19.84(13.80,25.87)^{\star}$ & $1.71(0.70,2.72)^{\star}$ & $2.33(1.26,3.40)^{\star}$ \\
IL-4 & $-13.50(-17.74,-9.26)^{\star}$ & $7.66(4.67,10.65)^{\star}$ & $-2.47(-4.78,-0.15)^{\star}$ \\
IL-17 & $28.92(17.44,40.40)^{\star}$ & $5.06(0.28,9.85)^{\star}$ & $-8.84(-33.52,15.83)$ \\
IL-23 & $310.60(4.96,616.24)^{\star}$ & $46.18(-7.60,99.97)$ & - \\
IL-10 & $-10.33(-12.03,-8.63)^{\star}$ & $-5.26(-9.53,-0.99)^{\star}$ & - \\
IL-6 & $160.71(-10.44,331.86)$ & $99.34(45.84,152.84)^{\star}$ & $18.93(-6.72,44.58)$ \\
IL-22 & - & $43.88(28.17,59.59)^{\star}$ & - \\
IL-8 & - & $0.02(-0.99,1.03)$ & $0.33(-0.57,1.22)$ \\
\hline
\end{tabular}

*, $\mathrm{P}<0.05 . \mathrm{MD}$ in immunological markers between BHS and controls according to a previous study (22). BHS, blood-heat syndrome; BSS, blood-stasis syndrome; BDS, blood-dryness syndrome; MD, mean difference; Cl, confidence intervals; IFN, interferon; IL, interleukin; TNF, tumor necrosis factor.

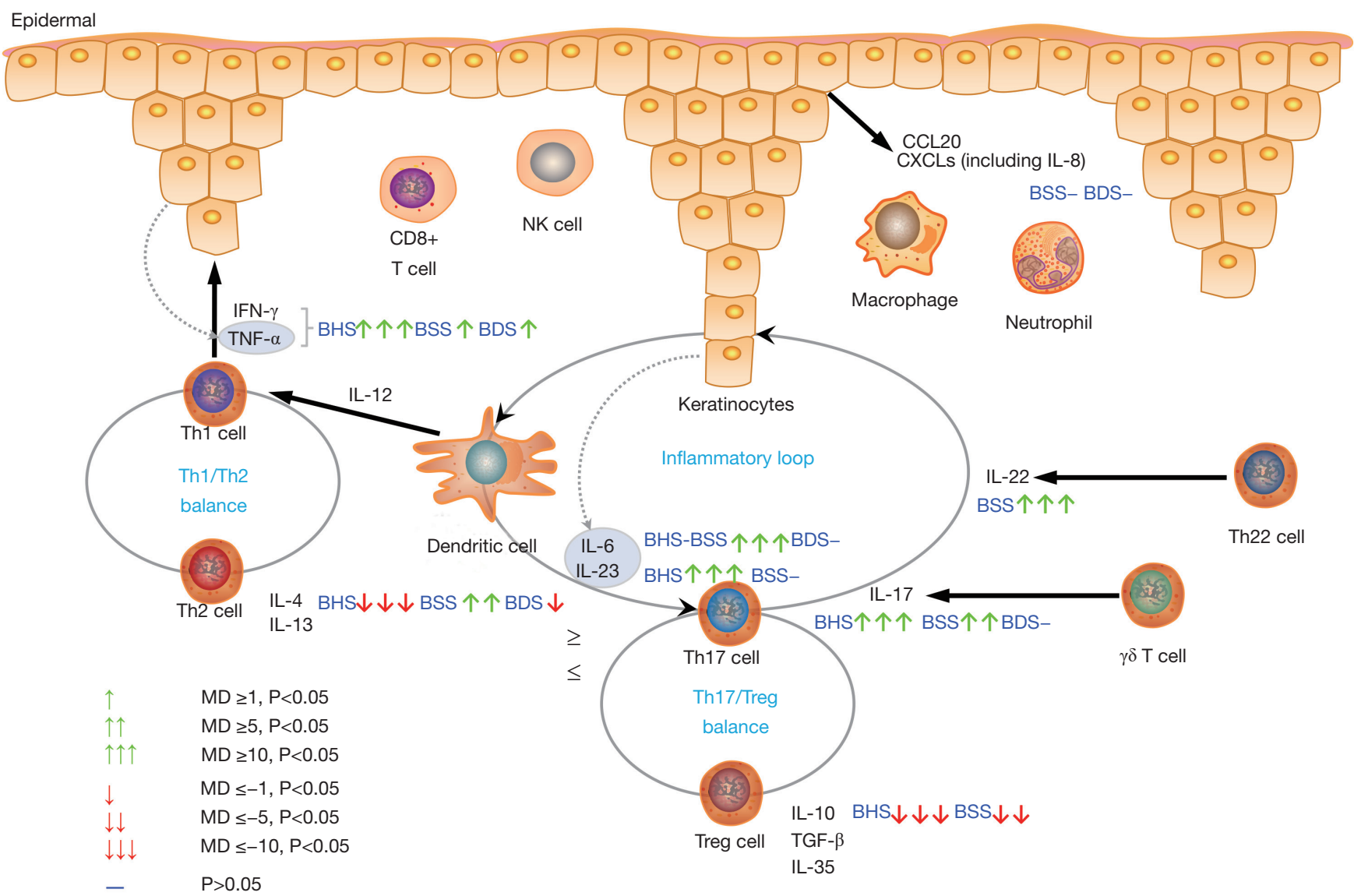

Figure 4 A simplified model representing the role of the inflammatory cytokines analyzed in psoriasis vulgaris of BHS, BSS, and BDS. IFN, interferon; IL, interleukin; Th, helper T; Treg, regulatory T; MD, mean difference; TGF, transforming growth factor. 
some ILs to produce a therapeutic effect (30). In addition, $\mathrm{CM}$ act on not only the IL monomer but also Th1/Th2 homeostasis, Th17/Treg balance, the IL-23/Th17 axis, and the related cytokines $(27,28,49,50)$.

\section{Limitations of this review}

Our analysis has some limitations. First, regarding the crosstalk between immune cells and cytokines involved in the immunological dysfunction in psoriasis, we were unable to analyze the abundance of immune cell types and the levels of additional relevant cytokines. Although we believe that our search strategy allowed us to find all relevant studies, there is still a degree of uncertainty. Furthermore, the 15 included studies must be cautiously interpreted, considering their small sample sizes and variability in the quality of the study design. Further larger, well designed, controlled studies are needed to confirm our results and fully clarify the alterations of the immune system in psoriasis.

\section{Conclusions}

Patients with psoriasis vulgaris of BSS show significantly elevated levels of IFN- $\gamma$, IL-4, IL-17, IL-6, TNF- $\alpha$, and IL-22, and decreased levels of IL-10, while those with psoriasis vulgaris of BDS show significantly higher levels of IFN- $\gamma$ and TNF- $\alpha$, and lower levels of IL-4. These inflammatory cytokines and several cell types are involved in the pathogenesis of psoriasis vulgaris of BHS, BSS, and BDS; in particular, Th1/Th2 homeostasis, Th17/Treg balance, and the IL-23/Th17 axis play important roles.

\section{Acknowledgments}

Funding: This study was supported by the National Science Foundation of China (NSFC, grant no. 81874470), National Key Research and Development Program of China (grant no. 2018YFC1705301), Shanghai Development Office of TCM [grant nos. ZY (2018-2020)-FWTX-1008, ZY (2018-2020)-CCCX-2004-08, ZY (2018-2020)-FWTX4010], Shanghai Municipal Health Commission (grant no. shslczdzk05001), and Young Talent Supporting Program of China Association of Traditional Chinese Medicine [grant no. CACM-2017-QNRC2-(B05)].

\section{Footnote}

Conflicts of Interest: All authors have completed the
ICMJE uniform disclosure form (available at http://dx.doi. org/10.21037/apm-19-432). The authors have no conflicts of interest to declare.

Ethical Statement: The authors are accountable for all aspects of the work in ensuring that questions related to the accuracy or integrity of any part of the work are appropriately investigated and resolved. This study was based on previously published studies; therefore, ethical approval and patient consent were not required.

Open Access Statement: This is an Open Access article distributed in accordance with the Creative Commons Attribution-NonCommercial-NoDerivs 4.0 International License (CC BY-NC-ND 4.0), which permits the noncommercial replication and distribution of the article with the strict proviso that no changes or edits are made and the original work is properly cited (including links to both the formal publication through the relevant DOI and the license). See: https://creativecommons.org/licenses/by-nc-nd/4.0/.

\section{References}

1. Parisi R, Symmons DP, Griffiths CE, et al. Global epidemiology of psoriasis: a systematic review of incidence and prevalence. J Invest Dermatol 2013;133:377-85.

2. Helmick CG, Lee-Han H, Hirsch SC, et al. Prevalence of psoriasis among adults in the U.S.: 2003-2006 and 20092010 National Health and Nutrition Examination Surveys. Am J Prev Med 2014;47:37-45.

3. Zheng Q, Sun XY, Miao X, et al. Association between physical activity and risk of prevalent psoriasis: A MOOSE-compliant meta-analysis. Medicine (Baltimore) 2018;97:e11394.

4. Dowlatshahi EA, Wakkee M, Arends LR, et al. The prevalence and odds of depressive symptoms and clinical depression in psoriasis patients: a systematic review and meta-analysis. J Invest Dermatol 2014;134:1542-51.

5. Vena GA, Vestita M, Cassano N. Psoriasis and cardiovascular disease. Dermatol Ther 2010;23:144-51.

6. Takeshita J, Wang S, Shin DB, et al. Effect of Psoriasis Severity on Hypertension Control: A PopulationBased Study in the United Kingdom. JAMA Dermatol 2015;151:161-9.

7. Li X, Kong L, Li F, et al. Association between Psoriasis and Chronic Obstructive Pulmonary Disease: A Systematic Review and Meta-analysis. PLoS One 2015;10:e0145221.

8. Li X, Miao X, Wang H, et al. Association of Serum Uric 
Acid Levels in Psoriasis: A Systematic Review and MetaAnalysis. Medicine (Baltimore) 2016;95:e3676.

9. Kurd SK, Troxel AB, Crits-Christoph P, et al. The risk of depression, anxiety and suicidality in patients with psoriasis: A population-based cohort study. Arch Dermatol 2010;146:891-5.

10. Luo Y, Ru Y, Zhao H, et al. Establishment of Mouse Models of Psoriasis with Blood Stasis Syndrome Complicated with Glucose and Lipid Metabolism Disorders. Evid Based Complement Alternat Med 2019;2019:6419509.

11. Tsai YC, Tsai TF. Anti-interleukin and interleukin therapies for psoriasis: current evidence and clinical usefulness. Ther Adv Musculoskelet Dis 2017;9:277-94.

12. Fleischer AB Jr, Feldman SR, Rapp SR, et al. Alternative therapies commonly used within a population of patients with psoriasis. Cutis 1996;58:216-20.

13. Chen Z, Zhou D, Wang Y, et al. Fire needle acupuncture or moxibustion for chronic plaque psoriasis: study protocol for a randomized controlled trial. Trials 2019;20:674.

14. Xing M, Yan X, Yang S, et al. Effects of moving cupping therapy for plaque psoriasis: study protocol for a randomized multicenter clinical trial. Trials 2020;21:229.

15. Ru Y, Yan XN, Yang SQ, et al. Oral Taodan granules for mild-to-moderate psoriasis vulgaris: protocol for a randomized, double-blind, multicenter clinical trial. Ann Transl Med 2019;7:488.

16. Chen X, Hong S, Sun X, et al. Efficacy of fish oil and its components in the management of psoriasis: a systematic review of 18 randomized controlled trials. Nutr Rev 2020. [Epub ahead of print].

17. Zheng Q, Jiang W, Sun X, et al. Total glucosides of paeony for the treatment of psoriasis: A systematic review and meta-analysis of randomized controlled trials. Phytomedicine 2019;62:152940.

18. Lv M, Deng J, Tang N, et al. Efficacy and Safety of Tripterygium Wilfordii Hook F on Psoriasis Vulgaris: A Systematic Review and Meta-Analysis of Randomized Controlled Trials. Evid Based Complement Alternat Med 2018;2018:2623085.

19. Luo Y, Ru Y, Sun X, et al. Characteristics of psoriasis vulgaris in China: a prospective cohort study protocol. Ann Transl Med 2019;7:694.

20. Koo J, Arain S. Traditional Chinese medicine for the treatment of dermatologic disorders. Arch Dermatol 1998;134:1388-93.

21. Tse TW. Use of common Chinese herbs in the treatment of psoriasis. Clin Exp Dermatol 2003;28:469-75.
22. Li X, Xiao Q-Q, Li F-L, et al. Immune Signatures in Patients with Psoriasis Vulgaris of Blood-Heat Syndrome: A Systematic Review and Meta-Analysis. Evid Based Complement Alternat Med 2016;2016:9503652.

23. Zhang XJ, Zhao Y, Gao DR, et al. Research on the relationship between the identification of disease and Th1/Th2 in psoriasis vulgaris. Chin J Lepr Skin Dis 2009;25:432-4.

24. Zhang L, Liu X, Wang LH, et al. Effect of Blood-treating Prescriptions on Serum IFN- $\gamma$, IL-6, and TNF- $\alpha$ of the Psoriasis Patients with Different TCM Syndromes. J Trad Chin Med 2010;51:1083-5+92.

25. Chen JG, Yang ZB, Zhu MF. Experimental Study on Blood-Stage Treatment on Influencing IL-17 and IL-23 in Patients with Psoriasis Vulgaris. Chin Arch Trad Chin Med 2012;30:306-7.

26. Tang HS. The clinical study of Yujinxiaoyintang treating psoriasis of Qi-stagnated and blood stasis type and study of influence on serum levels of IL-4 and IL-22. Shandong University of Traditional Chinese Medicine. 2013.

27. Wu J, Wang WQ, Wu AW. Research on the imbalance of Th17/Treg cells in psoriasis patients of blood heat syndrome and blood stasis syndrome. Chin J Lepr Skin Dis 2013;29:446-8.

28. Chen J, Cao XX, Xu R, et al. Research on different expressions of peripheral blood Th1/Th2 cells in psoriasis patients of blood heat syndrome and of blood stasis syndrome. Chinese Journal of Integrated Traditional and Western Medicine 2014;34:46-50.

29. He X, Liu J, Xu P, et al. Effect of blood-stage treatment on cytokine IFN- $\gamma$, IL -4, IL-10 and IL-17 of patients with psoriasis vulgaris in the periods. International J Trad Chin Med 2014;36:692-4.

30. Fan B, Li X, Ze K, et al. Expression of T-helper 17 cells and signal transducers in patients with psoriasis vulgaris of blood-heat syndrome and blood-stasis syndrome. Chin J Integr Med 2015;21:10-6.

31. Hu XY, Yang WX. The correlation between serum TNF- $\alpha$, IFN- $\gamma$ and IL-4 and the syndrome of Traditional Chinese Medicine in psoriasis vulgaris. Journal of Military Surgeon in in Southwest China 2015;17:156-8.

32. Li LL. Study on the association mechanism of syndrome differentiation of psoriasis from the differentiation of $\mathrm{T}$ helper cells. Beijing University of Chinese Medicine, 2015.

33. Xuan ML, Lu CJ, Han L, et al. Circulating levels of inflammatory cytokines in patients with psoriasis vulgaris of different Chinese medicine syndromes. Chin J Integr Med 2015;21:108-14. 
34. Jiang CY, Wang JS, Li YW, et al. Yangxue Huaban Decoction in Treating Blood Dryness Syndrome of Plaque Type Psoriasis. Chinese Journal of Experimental Traditional Medical Formulae 2015;21:159-63.

35. Sun HW, Liang M, Cheng Y. Relationship between the Identification of Diseases and the Expression of IL-8 and CXCR-4 in Psoriasis Vulgaris. Chinese Journal of Dermatovenereology of Integrated Traditional and Western Medicine 2016;15:279-81.

36. Wu Q. The observation of Taohongsiwu Decoction Combined with NB-UVB in treatment of blood stasis type psoriasis and effects on IL-4 and IL-22. Shandong University of Traditional Chinese Medicine, 2016.

37. Long JW, Pi XM, Tu YT. Study on Serum Levels of Cytokines in Patients with Psoriasis Vulgaris of Different Chinese Medicine Syndromes. Journal of Basic Chinese Medicine 2017;23:234-6.

38. Stang A. Critical evaluation of the Newcastle-Ottawa scale for the assessment of the quality of nonrandomized studies in meta-analyses. Eur J Epidemiol 2010;25:603-5.

39. Stroup DF, Berlin JA, Morton SC, et al. Meta-analysis of observational studies in epidemiology: a proposal for reporting. Meta-analysis Of Observational Studies in Epidemiology (MOOSE) group. JAMA 2000;283:2008-12.

40. Zhang GZ, Wang JS, Wang P, et al. Distribution and development of the TCM syndromes in psoriasis vulgaris. J Tradit Chin Med 2009;29:195-200.

41. Abdallah MA, Abdel-Hamid MF, Kotb AM, et al. Serum interferon-gamma is a psoriasis severity and prognostic marker. Cutis 2009;84:163-8.

42. Onderdijk AJ, Baerveldt EM, Kurek D, et al. IL-4 Downregulates IL-1beta and IL-6 and Induces GATA3

Cite this article as: Yan JT, Wang QG, Liu XQ, Li B, Ru Y, Hong S, Sun XY, Liu M, Li X. The immune status of patients with psoriasis vulgaris of blood-stasis syndrome and blooddryness syndrome: a qualitative evidence synthesis. Ann Palliat Med 2020;9(4):1382-1395. doi: 10.21037/apm-19-432 in Psoriatic Epidermal Cells: Route of Action of a Th2 Cytokine. J Immunol 2015;195:1744-52.

43. Stockinger B, Veldhoen M. Differentiation and function of Th17 T cells. Curr Opin Immunol 2007;19:281-6.

44. Campbell DJ, Koch MA. Phenotypical and functional specialization of FOXP3 + regulatory T cells. Nat Rev Immunol 2011;11:119-30.

45. Deng S, May BH, Zhang AL, et al. Plant extracts for the topical management of psoriasis: a systematic review and meta-analysis. Br J Dermatol 2013;169:769-82.

46. Deng S, May BH, Zhang AL, et al. Topical herbal formulae in the management of psoriasis: systematic review with meta-analysis of clinical studies and investigation of the pharmacological actions of the main herbs. Phytother Res 2014;28:480-97.

47. Zhang CS, Yang L, Zhang AL, et al. Is Oral Chinese Herbal Medicine Beneficial for Psoriasis Vulgaris? A Meta-Analysis of Comparisons with Acitretin. J Altern Complement Med 2016;22:174-88.

48. Zhang LX, Bai YP, Song PH, et al. Effect of Chinese herbal medicine combined with acitretin capsule in treating psoriasis of blood-heat syndrome type. Chin J Integr Med 2009;15:141-4.

49. Cao XX, Xu R, Li FL, et al. The expression of Th1/ Th2/Th17 in peripheral blood of the patients with blood-heat syndrome of psoriasis. Chin J Lepr Skin Dis 2014;30:524-6.

50. Li FL, Xu R, Zeng QC, et al. Tanshinone IIA Inhibits Growth of Keratinocytes through Cell Cycle Arrest and Apoptosis: Underlying Treatment Mechanism of Psoriasis. Evid Based Complement Alternat Med 2012;2012:927658. 